\title{
El cuerpo vivo y la subjetividad trascendental en la fenomenología de Edmund Husserl
}

\author{
RUBÉN SÁNCHEZ MUÑOZ* \\ Universidad Popular Autónoma del Estado de Puebla (México) \\ ruben.sanchez.munoz@upaep.mx \\ JORGE MEDINA DELGADILlO** \\ Universidad Popular Autónoma del Estado de Puebla (México) \\ jorge.medina@upaep.mx
}

\begin{abstract}
Resumen
En este trabajo se explora el problema del cuerpo vivo en la fenomenología trascendental de Edmund Husserl y el entrelazamiento que tiene con la conciencia trascendental. Para ello se exploran diversas capas o momentos del tema. Primero: la justificación de la ausencia de un tratamiento del cuerpo en Ideas I debido a su enfoque estático. Segundo: el problema propiamente dicho de la constitución del cuerpo vivo en Ideas II desde una fenomenología genética. Tercero: la posibilidad de una ética de la corporalidad a partir del análisis de las ubiestesias y la constitución de ciertos valores. Cuarto: que el cuerpo representa un problema metafísico o que su tratamiento puede ser enfocado desde la metafísica, ya que se trata del cuerpo y la subjetividad trascendental como factum del mundo.
\end{abstract}

Palabras clave: cuerpo propio, subjetividad trascendental, fenomenología trascendental, ubiestesias, valor, metafísica.

\section{The living body and transcendental subjectivity in the phenomenology of Edmund Husserl}

\begin{abstract}
In this work, we explorer the problem of the living body in the transcendental phenomenology of Edmund Husserl and the interlacing that it has with transcendental conscience. To do this, we present several layers or moments of the theme. First, the justification of the absence of body in Ideas I due to their static approach treatment. Second, the problem of the constitution of the living body in Ideas II from a genetic phenomenology. Third, the possibility of an ethic of physicality (or the living body) from analysis of the ubiestesias and the constitution of the values. Quarter, that the living body represents a metaphysical problem, because the living body and the transcendental subjectivity are facts (factum) in the world.
\end{abstract}

Key words: living body, transcendental subjectivity, transcendental phenomenology, ubiestesias, value, metaphysics.

* Doctor en Filosofía por la Universidad Veracruzana. Profesor Investigador de la Facultad de Filosofía de la Universidad Popular Autónoma del Estado de Puebla (UPAEP) y miembro del Sistema Nacional de Investigadores del CONACYT. Es autor de Introducción al personalismo de Edith Stein (2016).

** Doctor en Filosofía por la Universidad Panamericana. Profesor investigador de la Facultad de Filosofía de la Universidad Popular Autónoma del Estado de Puebla (UPAEP) y miembro del Sistema Nacional de Investigadores del CONACYT. 
INTRODUCCIÓN

La pregunta que va a orientar este trabajo es la siguiente: ¿qué lugar ocupa el cuerpo dentro de la fenomenología trascendental de Edmund Husserl? Se trata de una pregunta central que puede abordarse a partir de una descripción fenomenológica en la que tenemos experiencia de nuestro propio cuerpo vivo (Leib) - y en un segundo momento del cuerpo ajeno. $\mathrm{El}$ análisis lo realizaremos en cuatro momentos. En el primero vemos el problema del cuerpo en Ideas I y el entrelazamiento de la conciencia con el cuerpo; en un segundo momento expondremos el tema de la constitución del cuerpo propio desde la subjetividad trascendental recuperando los análisis de Husserl en Ideas II. En seguida, dedicamos unas páginas al esclarecimiento de las relaciones entre las ubiestesias y los valores con la intención de fundamentar una ética del cuerpo o más bien, de una ética que de despliega a partir de las ubiestesias. Después de ello, quiero mostrar cómo el cuerpo, al posibilitar la existencia de la conciencia trascendental en el mundo, la conciencia trascendental como factum, llega a convertirse en un problema metafísico y, finalmente, presento una breve conclusión. A pesar del lugar importante que ocupa el cuerpo en la quinta de las Meditaciones cartesianas, aquí seguimos otro camino y no hemos considerado las indicaciones de Husserl, las cuales incorporaremos en descripciones posteriores.

\section{EL PROBLEMA DEL CUERPO EN IDEAS I}

La pregunta de la que estamos partiendo como punto de apoyo puede abordarse desde distintos planos. En primer lugar, hay que decir que el cuerpo no ocupa un lugar central en Ideas I de 1913. En esta obra fundamental, Husserl se propone realizar una introducción a la fenomenología pura. "Pura" (reinen) quiere decir que en ella no deben interferir elementos ajenos a la conciencia, que el sentido de la conciencia que allí se trata no es la conciencia de un individuo psicofísico particular o concreta, que la fenomenología pura "se ocupa de la "conciencia", de todas las especies de vivencias, actos y correlatos de actos" (Husserl, 2013: 79 [Hua III/I, 5]) ${ }^{1}$ y que se trata por tanto de la conciencia en general (o de la esencia (wesen) de la conciencia).

Dado que uno de los problemas que Husserl denuncia en la epistemología es el problema de la trascendencia, en un análisis fenomenológico

\footnotetext{
1 Las referencias a las obras de Husserl las citamos en primer lugar indicando la traducción y después entre corchetes el tomo de Husserliana y la página como Hua. En algunos casos solo se cita el tomo de husserliana y el número de página.
} 
como el que desarrolla y expone en Ideas I, las trascendencias deben ser justificadas y clarificadas a partir del método de la reducción. Entre las trascendencias se halla el mundo con todas las cosas materiales que lo constituyen, pero también se halla el ser humano visto como un individuo psicofísico, como un hecho o ser humano empírico; el ser humano y su cuerpo, son una trascendencia que debe ser esclarecida y descrita a partir de sus elementos constitutivos desde la fenomenología trascendental. No es exagerado decir que se trata de una gran dificultad. Visto desde la actitud natural, el hombre es un "objeto real como otros en el mundo natural" (Husserl, 2013: 148 [Hua III/I, 67]). Tiene que ponerse entre paréntesis y no darse por hecho ninguno de los resultados de las ciencias. En el esfuerzo de Husserl por esclarecer la esencia de la conciencia en esta obra y el descubrimiento de la conciencia como residuo fenomenológico, esto es como "una región de ser sui generis por principio", deja de lado el gran problema de saber si esa conciencia es una conciencia en un cuerpo o no, más bien, aunque este problema lo anuncia, como veremos más adelante, no propone una solución y se queda abierto; deja también la duda de si el sujeto trascendental y el yo puro tienen un cuerpo o no; pero de ello mismo se derivan más problemas, tales como la pregunta de si el ser humano empírico tiene alguna relación con el yo puro y el sujeto trascendental o no y, por tanto, queda la duda de saber si el sujeto trascendental que descubre la reducción trascendental es el ser humano, el hombre empírico o no lo es. De allí podrían derivarse muchos más problemas.

Estas cuestiones no se resuelven en Ideas I, pero el filósofo moravo anticipa algunas líneas que va a explorar más tarde. En el parágrafo 53 Husserl dedica una importante aproximación al tema del cuerpo y de su enlazamiento (Verknüpfung) con la conciencia (Bewusstsein). En efecto, Husserl se refiere a este entrelazamiento al hablar de los seres animados y la conciencia psicológica y dice que se trata de una importante "ampliación de los límites" (Husserl, 2013: 200 [Hua III/I, 116]) de las consideraciones fenomenológicas. El problema de fondo de estas reflexiones está en la postura de Husserl según la cual entre la conciencia y la realidad "se abre un verdadero abismo de sentido" (Husserl, 2013: 189 [Hua III/I, 105]). Con el fin de mostrar después lo relevante que es esta distinción en la fenomenología trascendental entre conciencia y realidad y el sentido metafísico que llegará a ocupar el cuerpo, citaré las palabras que siguen donde Husserl afirma que la realidad se refiere a "un ser que se matiza, que nunca puede darse absolutamente, meramente contingente y relativo" y que la conciencia es "un ser necesario y absoluto, que por principio no puede darse por medio de matización y aparición" (Husserl, 2013: 189 [Hua III/I, 105]). En la perspectiva de la fenomenología estática que Husserl expone en Ideas I, tanto el mundo espacio-temporal en el que se encuentra 
el hombre y el ser humano vistos como realidades, incluido aquí el cuerpo, solo pueden tener un sentido intencional relativo a la conciencia dice Husserl, y ello significa que su sentido es secundario, que se trata de un ser "que la conciencia pone en sus experiencias", algo que ciertamente se da y de lo cual hay experiencia pero detrás de lo cual no hay nada o "es una nada" (Husserl, 2013: 190 [Hua III/I, 106]).

La conciencia, como hemos visto, es considerada como un ser absoluto y en ella se constituye todo lo trascendente. Lo trascendente solo tiene un sentido correlativo a la conciencia, sencillamente decir que lo trascendente es aquello que está más allá de la conciencia o que es independiente de ella es algo que carece de sentido (Husserl, 2009: 25 [Hua I, 24s]). Por ello, la constitución se refiere a la dación o donación de sentido en la conciencia pura.

Pues bien, en este parágrafo de Ideas I que estamos analizando, Husserl esboza un problema de conciliación. Se trata de conciliar la conciencia y la realidad. ¿Cómo conciliar que el ser absoluto de la conciencia -que en su descripción trascendental se revela como no siendo una parte del mundo, como un ser anterior al mundo-y, por otro lado, el hecho de que la conciencia entra en el mundo real y se convierte en una trascendencia? Esto solo puede hacerlo, a decir de Husserl, a partir de la participación de la conciencia en la trascendencia, o sea apareciendo ella misma en el mundo, materializándose podríamos decir. Aquí dice Husserl lo siguiente:

Solo por su referencia de experiencia con el cuerpo se convierte la conciencia en realmente humana y animal, y solo por ello obtiene un lugar en el espacio de la naturaleza y en el tiempo de la naturaleza... solo mediante el enlace de la conciencia y el cuerpo en una unidad empíricamente intuitiva, natural, es posible algo así como una comprensión mutua entre los seres animales pertenecientes a un mundo, y que solo por ello cada sujeto cognoscente puede hallar el mundo en su plenitud, consigo y con los otros sujetos y a la vez reconocerlo como el mismo mundo circundante, perteneciente en común a él y a todos los demás sujetos (Husserl 2013: 201 [Hua III/I, 116s]).

Pues bien, en este parágrafo Husserl expone que es gracias a este "entrelazamiento" como se da la "realización de la conciencia"; pero que aparezca en el mundo y que se materialice en un cuerpo no implica que la conciencia misma se da o aparece por medio de matizaciones o escorzos, como ocurre con las demás cosas materiales. La materialidad de la conciencia, que es el cuerpo, necesitará un profundo esclarecimiento por parte de Husserl. Él afirma que la conciencia al materializarse en un cuerpo se constituye como una trascendencia, aunque en un sentido peculiar, pero 
de allí, sin esclarecer aún su sentido, afirma que en este aparecer (o fenómeno) se da de manera particular la aparición (Ercheinung) de "un ESTADO de conciencia de un sujeto-yo real idéntico, que en dicho estado manifiesta sus PROPIEDADES REALES INDIVIDUALES y que ahora es conciente... como unido con el cuerpo que aparece" (Husserl, 2013: 201s [Hua III/I, 117]).

Ahora bien, el descubrimiento de estas propiedades reales individuales va a tener un gran alcance en Ideas II y serán fundamentales para la comprensión del sentido de la persona humana y, en especial, para la comprensión de este entrelazamiento entre la conciencia y el cuerpo y el sentido corporal de la subjetividad trascendental a través de la persona y, también, para la superación del yo puro impersonal y sin cualidades, y el paso al yo personal como subjetividad trascendental corporal e histórica - de la que hablaremos en el siguiente apartado. Pero en Ideas I Husserl no anticipa nada de ello y esta es la razón por la cual uno podría llegar a pensar que el sujeto trascendental es una mera forma vacía que no se refiere a la realidad humana concreta como le pasó a José Gaos (1997). En efecto, en el congreso que se celebró en México en 1963 en el marco del XIII Congreso Internacional de Filosofía donde se realizó el "Simposio sobre la noción Husserliana de Lebenswelt", en el que estuvieron Enzo Paci, Ludwig Landgrebe, John Wild, Luis Villoro y el mismo Gaos, éste último sostuvo una idea del sujeto trascendental como "único y abstracto" (San Martín, 2015a: 53). Además de ello, Gaos defendió la idea del cuerpo que aquí hemos expuesto, la idea del cuerpo como un "objeto constituido" por el sujeto trascendental, idea que por lo demás es parcial. A decir de San Martín, Gaos entiende el sujeto trascendental como este sujeto que remite a lo "ideal conceptual", de lo cual se sigue que el sujeto trascendental no puede tener un cuerpo; el sujeto trascendental en el que piensa Gaos lo resume San Martín diciendo que se trata de "un sujeto trascendental incorpóreo, por ser ideal, abstracto, intemporal y ahistórico" (2015a: 59).

En los análisis que realiza Husserl en Ideas II sobre el cuerpo se deja ver el error de Gaos al respecto y el lugar que ocupa el cuerpo en la fenomenología trascendental de Husserl, lo cual no quiere decir que se despejen todas las dudas, pero se muestra con ello que el sujeto trascendental es un sujeto encarnado, con una historia y una vida humana concreta que despliega teleológicamente en una comunidad, en relación intersubjetiva con los otros, y no de manera solipsista y aislada como podría pensarse en un primer momento. Puede decirse que el mundo para Husserl no es solo el mundo del ego sino un mundo en común, esto es, un mundo que es constituido intersubjetivamente. No vivimos el mundo de manera solipsista, sino que es compartido por otros con los cuales mantenemos relaciones en distintos planos. Aunque es difícil afirmar la presencia del alter 
ego mediante la vía cartesiana que sigue Husserl en las Conferencias de París y en la quinta de las Meditaciones cartesianas, lo cierto es que Husserl dedica importantes trabajos al problema de la intersubjetividad y descubre la subjetividad trascendental como intersubjetividad trascendental, en la que la posibilidad del sujeto trascendental individual aislado se desvanece. Cabe decir que el cuerpo constituye uno de los ejes centrales para el análisis de la intersubjetividad en Husserl y uno de los puntos de acceso es justamente a través de la empatía. ${ }^{2}$ Pero, para decirlo con Merleau-Ponty, en Husserl mismo la intersubjetividad aparece como intercorporeidad, es decir, como sujetos cuya presencia corporal está dada de manera originaria, como habría mostrado ya Edith Stein en su tesis doctoral Sobre el problema de la empatía al hablar de la constitución del individuo extraño a partir de la experiencia corporal y esto es así justo porque la primera noticia que tenemos del otro es su cuerpo (Stein, 2004).

Ahora bien, aunque el tema del cuerpo da para un análisis de la intercorporeidad y de cómo el otro se aparece como otro en un cuerpo vivo, lo que aquí nos interesa no es la constitución del individuo extraño o del cuerpo vivo ajeno sino la experiencia (Erfharung) o vivencia (Erlebnis) del cuerpo vivo propio (Leib), que es justamente lo que vamos a realizar en el siguiente apartado.

\section{LA CONSTITUCIÓN DEL CUERPO PROPIO EN IDEAS II}

Lo primero que hay que decir es que el análisis del cuerpo que Husserl realiza en Ideas II y el cual vamos a exponer aquí, es un análisis desde una perspectiva trascendental, desde la subjetividad trascendental conseguida por la reducción y en segundo lugar es preciso señalar que a diferencia de las descripciones que realiza Husserl en Ideas I las realiza desde una fenomenología estática, en Ideas II se trata más bien de una fenomenología genética. Si bien en la fenomenología estática se analiza la vida trascendental como algo acabado a partir de la descripción de los modos de aparecer los objetos en la conciencia y las operaciones subjetivas que posibilitan su constitución, en el análisis genético se añade, como señala Walton, "el análisis de la constitución o autodesarrollo de la vida trascendental" (Walton, 2015: 46). Además, el análisis genético permite examinar la génesis de la constitución a partir del despliegue histórico de la conciencia y el análisis

2 En el parágrafo 5 del Anexo XII de Ideas II, Husserl habla de la empatía como "referencia espiritual (no-natural) entre sujetos" y dice que "El cuerpo ajeno es para mí pasaje (en la "expresión", en la inclinación, etc.), para la comprensión del otro allá, del él; él mueve la mano, él coge esto o aquello, él da un golpe, él piensa, es motivado por esto y aquello" (Husserl, 2005: 400 [Hua IV, 347]). 
de las formaciones precedentes y subsiguientes de la vida de la conciencia; así, "se contempla la historia de los actos y sus objetos mediante preguntas retrospectivas por las distintas implicaciones intencionales de orden temporal" (Walton, 2015: 46).

Pues bien, el aspecto importante de Ideas II respecto del cuerpo lo encontramos de manera sustancial en la segunda sección, donde aborda tres cuestiones fundamentales, a saber: la materia, la animalidad y la espiritualidad. ¿Qué relación mantiene el ser humano con ellas y qué lugar ocupa el cuerpo para la comprensión de estas instancias? Ideas II aborda el tema que Husserl puso entre paréntesis en el parágrafo 53 de Ideas I: la constitución del mundo animal. Si en Ideas I da la impresión que el mundo del que habla Husserl no es el mundo humano, porque de alguna manera ha puesto entre paréntesis la realidad del ser humano empírico y el sentido del mundo como mundo de la cultura (o mundo espiritual), o del mundo como mundo intersubjetivo donde se despliega la vida personal y comunitaria de las personas humanas, en Ideas II se trata justamente de eso, de abordar el mundo de las personas y el sentido humano del mundo humano. Y Husserl empieza hablando del yo puro como polo-yo en el parágrafo 22 para pasar más adelante a mostrar la importancia de la realidad anímica y del lugar intermedio que ocupa la vida en medio de la materia y el espíritu. En una serie escalonada, lo que tenemos es realidad material, realidad anímica (o vida animal que forma parte también de la realidad humana) y realidad espiritual. Por vida anímica, hay que decir que Husserl comprende la vida animal, la que podemos sintetizar en la sensibilidad. Por ello el cuerpo vivo o propio ocupa un lugar central en esta obra. Aquí no podemos exponer el sentido de cada una de estas instancias, nos vamos a centrar de manera puntual en la descripción del cuerpo.

Husserl distingue el yo puro o trascendental del sujeto anímico real o el alma. Por ella entiende el "ser psíquico idéntico" que está vinculado realmente - dice-con el cuerpo humano o animal. Por ello, como vamos a ver, lo que Husserl llama alma (Seele) en esta obra se refiere más bien a la psique que está enlazada con el cuerpo. Este sujeto entendido como realidad anímica se distingue del yo puro por ser el portador de propiedades personales. Este sujeto personal, además de poseer sus vivencias propias, posee un cuerpo, pero se trata de un cuerpo material que él anima, en el cual él vive y tiene vivencias corporales; el sujeto es uno con su cuerpo. La vida humana o la realidad anímica animal tiene como característica fundamental la irreversibilidad, es decir, el hecho de ser una realidad animal histórica que se despliega en el tiempo. Esta tesis tendrá serias implicaciones desde el punto de vista del cuerpo como veremos.

Pues bien, el cuerpo vivo (Leib) como cuerpo anímico y cuerpo propio, es la realidad más inmediata que tenemos para empezar la descripción 
de su constitución o sentido y esto lo hace en el capítulo III de la segunda sección de Ideas II que Husserl dedica a la descripción de "La constitución de la realidad anímica a través del cuerpo". En efecto, las descripciones que encontramos en los parágrafos 36 al 38 de Ideas II sobre la constitución del Leib son fundamentales para comprender el tema que nos ocupa, en especial porque el cuerpo vivo funge como instancia intermediaria entre la realidad material y la espiritual. El cuerpo propio ni es completamente naturaleza ni es completamente espíritu, sino una unidad peculiar constituida por elementos que conciernen a ambas esferas. En estos pasajes Husserl describe, como pocas veces lo hace, tres experiencias corporales de vital importancia: las experiencias de la mano, las de la vista y el oído y las del cuerpo como órgano de la voluntad. Lo más importante de sus análisis y lo cual podemos empezar a enumerar son los siguientes resultados:

En primer lugar, que el cuerpo vivo se constituye en una doble dirección. Por un lado, es un cuerpo material o físico (Körper) y en este sentido el cuerpo pertenece al ámbito de la naturaleza material. El cuerpo tiene las mismas cualidades que otro cuerpo en el espacio, responde a las leyes de la física, etc. Solo que no es solo eso. Es un cuerpo vivo o propio (Leib). Husserl emplea una estructura tripartita para describir al ser humano, y ello es fundamental para hablar de la antropología fenomenológica de Husserl. Esta estructura está conformada por cuerpo/psique/espíritu y, como puede verse, aparece el cuerpo justamente ocupando un lugar importante no solo como cuerpo geométrico sino como cuerpo personal. De acuerdo con ello, podríamos anticipar tres perspectivas distintas que encontramos en la realidad humana: una material o física a la que accedemos y aceptamos solo a partir de un proceso de abstracción como la que se da en la actitud naturalista; la segunda se refiere al cuerpo vivo como realidad animal inmediata y, finalmente el hombre como espíritu, como creador de cultura y valores, instituciones, etc. Todo ello había quedado suspendido o puesto entre paréntesis en Ideas I, por ejemplo, todo el mundo de la cultura y de los valores y el sentido animal de la percepción y el carácter dinámico de la constitución del mundo (Husserl 2013: 144s [Hua III/I, 65s]), de tal modo que el mundo del que se habla en Ideas I no es el mundo humano, sino también una especie de abstracción al que se accede a partir de la modificación de la actitud primigenia de la vida ante el mundo circundante. Esta actitud primigenia de la vida humana en el mundo circundante (Umwelt), no es la naturalista científica ni tampoco la actitud filosófica, sino la actitud que Husserl llama personalista.

El aspecto que permite resaltar la doble constitución del cuerpo lo ejemplifica Husserl a partir del cuerpo como el portador de sensaciones localizadas que designa como ubiestesias (Empfindniss). En el parágrafo 36 
afirma que todas las sensaciones ocasionadas "tiene su localización, es decir, se diferencian por los sitios de la corporalidad aparente y pertenecen fenomenalmente a ella". En este mismo lugar sostiene que el cuerpo se constituye "primigeniamente de manera doble: por un lado, es cosa física, MATERIA... por otro lado, encuentro en él, SIENTO “en" él y "dentro" de él" (Husserl, 2005: 185 [Hua IV, 145]).

$\mathrm{Al}$ entrar el cuerpo en contacto con el entorno material se experimenta en esta doble dirección, si bien no siempre la mirada está dirigida a ambos lados. Hay además una segunda capa primigenia que Husserl presenta a través del análisis de la experiencia táctil, en especial se trata de la experiencia del cuerpo que se experimenta o vivencia a sí mismo como cuerpo. Husserl lo denomina Leibkörper, un cuerpo vivo que es también cuerpo material. Es posible referirse a este fenómeno distinguiendo entre el cuerpo objeto y el cuerpo sujeto (San Martín, 2015a: 66). El cuerpo propio tiene experiencia de sí mismo, no solo experimenta cosas distintas que de hecho le afectan todo el tiempo, sino que él mismo se experimenta a través de las ubiestesias, como cuerpo vivo está atravesado por la vida sensible y por ello su vida es "vivida desde dentro" (San Martín, 2015a: 67). Las sensaciones localizadas son propiedades de la cosa cuerpo y se presentan justo cuando "el cuerpo es tocado, presionado, pinchado etc., y se presentan AHÍ DONDE lo es y CUANDO lo es" (Husserl, 2005: 186 [Hua IV, 146]).

En efecto, Husserl afirma que el cuerpo como campo de localización "es la presuposición para los demás distintivos del cuerpo frente a todas las cosas materiales". Así, la percepción de las cosas está ligada necesariamente a la percepción del cuerpo propio por varias razones, sobre todo porque nuestro cuerpo está siempre presente en nuestra percepción del mundo, en los actos perceptivos. No hay un lugar de la tierra en la que yo esté presente sin que esté presente mi cuerpo. El cuerpo va a donde yo voy. Y lo que le pasa a mi cuerpo me pasa a mí en persona, lo sufro y lo padezco, o lo gozo y disfruto. Esto es así debido a que el cuerpo propio se constituye como centro de orientación (Husserl, 2005: 197s [Hua IV, 158]), o lo que es lo mismo: "como punto cero del sistema de coordenadas" [Hua XIII, 116]. El cuerpo está esencialmente en un aquí originario que lo posiciona espacialmente y las cosas se exhiben en función de esta posición del cuerpo: "cada yo tiene su dominio perceptivo cósico, y necesariamente percibe las cosas en cierta orientación" (Husserl, 2005: 197 [Hua IV 158$])$. En otro lugar dice Husserl lo siguiente: "cada quien tiene su fenómeno de la cosa, y para cada uno la misma cosa aparece de otro modo según la posición espacial que ocupe" [Hua XIII, 117]. Las coordenadas espaciales solo tienen sentido a partir de nuestro cuerpo: arriba, abajo, izquierda, derecha, enfrente, detrás. Merleau-Ponty, que en este punto sigue fielmente a Husserl a nuestro juicio, enfatiza que soy yo, con mi cuerpo 
quien "observo los objetos exteriores... los manipulo, los examino, doy la vuelta a su alrededor" (Merleau-Ponty, 1975: 109).

Esto mismo lo desarrolla Husserl y de ello extrae otro elemento constitutivo del cuerpo propio que consiste justamente en el movimiento del cuerpo que Husserl desarrolla en el parágrafo 38 de Ideas II. En particular, Husserl sostiene allí que el distintivo del cuerpo como cuerpo vivo es justamente el de "ser ÓRGANO DE LA VOLUNTAD, el ÚNICO OBJETIVO que para la voluntad de mi yo puro es movible de manera inmediatamente espontánea y medio para producir un movimiento espontáneo mediato de otras cosas". (Husserl, 2005: 191 [Hua IV, 151s]). El movimiento del cuerpo propio influye de manera directa en el modo como las cosas se aparecen. Como órgano de la voluntad y portador de movimiento, el cuerpo vivo se convierte en el medio para realizar cualquier otra actividad en el mundo; con el movimiento del cuerpo se modifica inmediatamente la percepción del entorno cósico, posibilita que las cosas muestren aspectos o escorzos que una perspectiva anterior no me daba. El cuerpo de presenta como contramiembro de la naturaleza a través de un yo que ejecuta actos libres.

Estos actos libres son aquellos gracias a los cuales... puede constituirse para este yo en múltiples series de percepciones un mundo de objetos, un mundo de cosas corpóreas espaciales... El sujeto que se constituye como contramiembro de la naturaleza material es... un yo al cual le es inherente un cuerpo como campo de localización de sus sensaciones; tiene la "capacidad" ("yo puedo") para mover libremente este cuerpo o los órganos en que se articula, y para percibe mediante ellos un mundo externo (Husserl 2005: 191s [Hua IV, 152]).

Dice San Martín que "las cinestesias, con las que está íntimamente conectada la visión del cuerpo real, pertenecen a la subjetividad trascendental" (San Martín, 2015a: 48) y por esta razón hay que sostener que a través de las cinestesias la subjetividad tiene una relación estrecha con el cuerpo, más aún: que la subjetividad trascendental se constituye corporalmente. En palabras de Waldenfels (2015: 177s) hay que sostener que habitamos "corporalmente en el espacio" y que la constitución del mundo cósico no está separada de este modo de ser corporal. En efecto, los análisis que realiza Husserl sobre la experiencia táctil, nos muestra cómo en cuerpo vivo se desdobla y se experimenta a sí mismo como cuerpo vivo sentiente que siente, que se vivencia a sí mismo a través de las sensaciones localizadas (Empfindniss). Pero esta es una forma de ser esencial de la constitución del cuerpo a partir de las sensaciones táctiles. La mano ocupa un lugar privilegiado respecto del ojo y el oído. El órgano corporal que toca 
puede tocarse y de este modo llegar a adquirir una consistencia que puede describirse a partir de una doble intencionalidad. Husserl afirma que "mi cuerpo en cuanto cuerpo palpado es algo palpado que palpa" (Husserl, 2005: 187 [Hua IV, 148]). Así, las ubiestesias permiten distinguir al cuerpo vivo (Leib) de cualquier otro cuerpo material (Körper). El cuerpo solo se convierte o constituye en cuerpo vivo "mediante la introducción de las sensaciones en el palpar, mediante la introducción de las sensaciones en el dolor, etc., en suma, mediante la localización de las sensaciones en cuanto sensaciones" (Husserl, 2005: 191 [Hua IV, 151]). Las sensaciones localizadas son, por ello, el "distintivo específico" del cuerpo vivo en cuanto cuerpo vivo, y esto es así debido a que el valor que tiene las sensaciones táctiles para la constitución del cuerpo no la tiene el campo de las sensaciones visuales o auditivas. La mano palpa y es palpada, siente y se siente. Pero la vista no se ve a sí misma y el oído no se escucha a sí mismo y por ello no se puede hablar de ubiestesias visuales o auditivas, pero sí podríamos hablar de las ubiestesias en el cuerpo en general, en el cuerpo entendido como carne -pero no podemos desarrollar esta línea en este momento. En las experiencias táctiles nos encontramos con sensaciones dobles $^{3}$. Estas ubiestesias son mías, aparecen en mi cuerpo vivo y no se dan en los cuerpos no vivos; ellas forman parte de mi conciencia y de mi vida y, por tanto, forman parte de mi alma. Son las ubiestesias las que le permiten a Husserl hablar en Ideas II nuevamente del entrelazamiento entre el cuerpo y el alma: "lo que es aprehensible como estrato localizado del cuerpo, y más, lo que es aprehensible como dependiente del cuerpo... y de los "órganos sensoriales", todo ello compone, bajo el título de materia de la conciencia, un subsuelo de la conciencia y experimenta su aprehensión realizadora a una con ésta en cuanto alma y yo anímico" (Husserl, 2005: 196s [Hua IV, 157]). Husserl concluye el parágrafo 40 de Ideas II afirmando que el alma y el yo anímico tiene un cuerpo:

[...] existe una cosa material de cierta naturaleza, que no es meramente cosa material, sino cuerpo, o sea: una cosa material que como campo de localización de sensaciones y de mociones afectivas, como complejo de órganos

\footnotetext{
3 Merleau-Ponty cita el tomo segundo de Ideas en su Fénoménologie de la perception de 1945 y desarrolla estos temas cuando la obra aún no se publicaba gracias a que tuvo acceso a los manuscritos inéditos de Husserl (Merleau-Ponty, 1975: 108s); pero hay que decir que Edith Stein, en un periodo muy temprano también recuperó aspectos importantes de Ideas II en sus trabajos, sobre todo porque ella trabajó en la edición de esta obra mientras fue asistente de Husserl en Friburgo. Este influjo de Husserl sobre Stein se deja ver en varios de sus trabajos, en especial en su libro Sobre el problema de la empatía.
} 
sensoriales, como miembro y contramiembro de todas las percepciones cósicas... compone un terreno fundamental de la dación real del alma y del yo (Husserl, 2005: 197 [Hua IV, 157]).

Pues bien, a pesar de que el cuerpo está presenta siempre y "porta en sí el PUNTO CERO de todas estas orientaciones", en un "aquí central último, esto es, un aquí que no tiene ningún otro fuera de sí en referencia al cual él fuera un 'allí' " (Husserl, 2005: 198 [Hua IV, 158]), a pesar de ello la constitución misma del cuerpo resulta bastante extraña. Esta cierta extrañeza del cuerpo propio la expone Husserl en el parágrafo 41, en el b), sin hacer mención allí de la extrañeza del cuerpo propio - como lo va a tematizar en varios escritos B. Waldenfels (2015). Husserl expone que esta libertad originaria del cuerpo propio para cambiar de posición, moverse y con ello constituir el mundo en una multiplicidad de apariciones,

[...] no tengo la posibilidad de alejarme de mi cuerpo o de alejarlo a él de mí, y en correspondencia con ello las multiplicidades de aparición del cuerpo están en determinada manera restringidas: ciertas partes del cuerpo solo puedo verlas en un peculiar acortamiento perspectivo, y otras (por ejemplo, la cabeza) son invisibles para mí. El mismo cuerpo que me sirve como medio de toda percepción me estorba en la percepción de sí mismo y es una cosa constituida de modo curiosamente imperfecto (Husserl, 2005: 199; [Hua IV, 159]).

Pues bien, hasta aquí podemos decir que el análisis de Husserl es un análisis constitutivo que se lleva a cabo describiendo la experiencia trascendental a partir de la cual la subjetividad trascendental se encuentra entrelazada con un cuerpo. La percepción del cuerpo vivo que Husserl describe mantiene un vínculo esencial con la psique - $\mathrm{O}$ alma. Por ello puede afirmar que el cuerpo está inserto entre el conjunto de cosas materiales y mantiene con ella relaciones causales que condicionan y determinan la vivencia del cuerpo y su constitución. Aunque esta sea interna o externa, lo que encontramos en ella es que el cuerpo vivo es un "punto de trasbordo", dice Husserl, "en el que las relaciones causales se traducen en relaciones condicionales entre el mundo externo y el sujeto anímico-corporal" (Husserl, 2005: 201 [Hua IV, 161]).

\section{NOTAS PARA UNA VALORACIÓN ÉTICA DEL CUERPO}

En el parágrafo 39 de Ideas II se encuentran contenidas una serie de ideas, en algunos casos poco claras y de difícil análisis, a partir de las cuales se puede intentar justificar una ética del cuerpo o la forma como el cuerpo 
sirve de "trampolín" para los "valores sensibles" y para los "valores espirituales" (Walton, 2014), lo que Husserl llama "objetividades superiores" (Husserl, 2005: 192 [Hua IV, 152]).

Ya vimos que las ubiestesias (Empfindniss) son sensaciones localizadas en el cuerpo vivo. Pero en este apartado de Ideas II -y luego en otros lugares como veremos- Husserl identifica otro tipo de ubiestesias que pertenecen a "SENSACIONES DE GRUPOS ENTERAMENTE DISTINTOS" (Husserl, 2005: 192 [Hua IV, 152]). Husserl los llama "sentimientos sensibles". Con ellos se refiere a las sensaciones de placer y dolor, e incluye allí el bienestar o malestar. Se trata de experiencias que de una u otra manera invaden y llenan el cuerpo o lo "destemplan", fatigan, incomodan, etc. Husserl se refiere a una tipología de vivencias en las que se da la constitución de valores como sus correlatos intencionales y que son análoga a las vivencias intencionales en las que se constituyen los objetos cósicos. Habla de las sensaciones que juegan un papel importante para los "actos de valoración" pero los cuales resultan de "difícil análisis y discusión". Está pensando en sensaciones que "forman el soporte material de la vida desiderativa y volitiva, sensaciones de contracción y relajación de la energía, sensaciones de inhibición, parálisis y liberación interiores, etc." (Husserl, 2005: 192 [Hua IV, 153]).

En su libro Antropología filosófica II, Javier San Martín dedica un apartado a la corporeidad humana y en él recupera las ideas expuestas por Husserl que acabamos de mencionar. Sobre las sensaciones localizadas, las ubiestesias, San Martín se refiere al igual que Husserl a las sensaciones táctiles que se localizan en el cuerpo, pero de allí San Martín - siguiendo en el fondo a Merleau-Ponty - sostiene que, precisamente, "todo mi cuerpo, mi piel, es el lugar de las sensaciones localizadas, que son todas las del tacto; mi ropa, la base en que me siento, el suelo que piso, todo me da un conjunto de sensaciones localizadas" (San Martín, 2015b: 70). Pues bien, valiéndose de una distinción hecha por Agustín Serrano de Haro (1997), llama sensaciones cinestésicas a todas aquellas sensaciones que se relacionan con el movimiento del cuerpo. Entre ellas se pueden mencionar el movimiento que se produce al cerrar los ojos, al estirar el cuello o los brazos, al bostezar o cualquier movimiento de la cabeza. A ese otro grupo de sensaciones que Husserl califica como sensaciones de "difícil análisis y discusión" les llama cenestésicas. Todas ellas nos permiten aprehender objetos de la experiencia, pero en particular las cenestesias nos permiten constituir objetos valorados, lo que Husserl llama en Ideas II "objetividades superiores".

Lo que aquí nos concierne es indagar cómo se da este paso de las ubiestesias, o en particular de las cenestesias, a la vida práctica del sujeto 
en su mundo circundante. De acuerdo con San Martín, para Husserl el cuerpo vivo no solo está dotado de los campos sensibles,

[...] sino que todo ese contenido, es un cuerpo práctico, intencional, que en sus movimientos anticipa la meta final. La originalidad de los movimientos ejecutados por el cuerpo es que anticipan directamente la situación final; su intención no prefigura un recorrido espacial más que para alcanzar la meta dada en su lugar; la intención es como un germen de movimiento que luego se desarrolla, aunque solo secundariamente, en el recorrido objetivo (San Martín, 2015b: 72).

Es un cuerpo práctico, cabe decir, porque yo lo puedo mover libremente.

Sobre este mismo tema, Roberto Walton tiene un artículo sobre las "Facetas de la corporalidad en la ética husserliana". El filósofo argentino toma como punto de partida la experiencia del yo puedo primigenio para mostrar justamente que "el cuerpo propio tiene la función de "punto de partida de toda praxis" (Walton, 2014: 239). En el parágrafo 39 de Ideas II, Husserl afirma que "LA CONCIENCIA TOTAL DE UN HOMBRE está ENLAZADA EN CIERTA MANERA CON SU CUERPO MEDIANTE SU SOPORTE HYLÉTICO” (Husserl, 2005: 193 [Hua IV, 153]). Pero advierte que, en este nivel, las vivencias intencionales "ya no están propiamente LOCALIZADAS, ni forman ya un estrato en el cuerpo" y agrega las vivencias intencionales (por ejemplo, la percepción o el pensamiento de algo), están "entretejidos" con el cuerpo y por ello "tienen realmente localización intuitivamente dada, no las intencionalidades, y solamente por transferencia hablamos de ellas como referidas al cuerpo o incluso como existentes en el cuerpo" (Husserl, 2005: 193 [Hua IV, 153]). Roberto Walton recupera el problema hylético para retroceder en una "indagación genética" y mostrar que en la constitución corporal del sujeto hay una "protoestructura configurada por un componente exclusivamente hylético, un sentir sensible configurado por el agrado o el desagrado que provoca —un interés o desinterés en el ser atraído por este material hylético-, y el movimiento kinestésico que lo motiva" (Walton, 2014: 239). La byle afecta directamente las sensaciones cinestésicas y cenestésicas, afecta con ello la vida de conciencia en un nivel que por ser muy bajo todavía no se relaciona con la intencionalidad, pero ello determina la afectividad y el sentir. Un objeto con el cual tenemos alguna relación, puede motivar cierto placer, agrado o desagrado desde un punto de vista somático.

Walton resalta la relación que mantiene el cuerpo con los valores sensibles y con los valores espirituales. Advierte que en el nivel más bajo se encuentran los valores sensibles, como el placer. Husserl advierte en Hua 
$\mathrm{XV}$, que "los valores hedonistas tienen su origen en el gozo, en última instancia en los sentimientos sensibles y se efectivizan siempre en el gozo" (Walton, 2014: 242). Y a pesar de que el hedonista está ya por encima del animal porque no vive el placer solamente como gozo, si se entrega solo a la consecución y búsqueda del placer sin buscar una meta más alta o un valor más alto, entonces no ha logrado imprimirle a la vida una "meta vital universal" y en un nivel superior se encuentran los valores vitales como la salud y la comodidad que luego Walton confronta con las ideas de Scheler y los valores vitales o valores del sentir vital (Werte der vitalen Fbülens) y el cuerpo como el medio efectivo para la realización de los valores del que habla Ricoeur. Claro está que aquellos valores de nivel inferior pueden legar a operar como "condición de posibilidad de los valores del amor" y cita a Husserl: "valores de gozo de nivel inferior pueden alcanzar mediatamente un valor de amor en razón de que son conocidos y apreciados como condición de posibilidad de valores de amor" (en Walton, 2014: 244).

La postura de Husserl sobre los valores, como muestra Walton, es que ellos "pueden alcanzar el carácter de mandamientos racionales como trampolines que hacen posibles futuros impulsos hacia actividades y producciones espirituales" (Walton, 2014: 246). Lo importante de los valores espirituales es que ellos están ligados a la persona y depende de ella, de sus cualidades personales, de su mundo de intereses y de las tomas de postura que asume. Un valor sensible puede no identificarse con la persona que lo goza, pero un valor espiritual que se vivencia en carne propia no puede no identificarse con la estructura personal —si es que se le puede llamar así. Mientras los valores sensibles dependen de manera directa de una "afección pasiva" los valores espirituales responden a una "producción espiritual". Configurarse como yo personal implica tomar decisiones y posturas frente al mundo, las cosas y los otros. Husserl habla de la autosatisfacción (Selbstzufriedenheit) como el centro de la felicidad (Glückseligkeit) y esta no se puede alcanzar más que asumiendo una "permanente disposición ética en acciones justificadas" (Walton, 2014: 246). Son justificadas aquellas acciones o comportamientos que responden a la legalidad racional de la vida, esto es, la motivación. El hombre vive como ser finito, pero en el horizonte de la infinitud. 'El hombre vive en la 'infinitud', que es su constante horizonte vital, sobrepasa los instintos, crea valores de nivel superior y sobrepasa estos valores" (Walton, 2014: 247).

En el fondo de este planteamiento el cuerpo ocupa un lugar importante y en especial la experiencia del "yo puedo" que experimenta el sujeto corporal. El supuesto es que el sujeto corporal "puede hacer" e inclusive dejar de hacer, frente a las irracionalidades que presenta la contingencia de la realidad fáctica. Mediante la asimilación de ciertos valores espirituales 
- como el amor o la búsqueda del bien- el sujeto ético puede enfrentarse a muchas dificultades e impedimentos que provienen del exterior, "sucesos fortuitos e incalculables que perturban la razón práctica" afirma Walton. Husserl habla en este contexto de "contradicciones entre sus objetivos humanos universales y las anomalías irracionales del mundo, con sus contingencias, con sus destinos a partir de la naturaleza y en la vida de la comunidad y la muerte que se presenta 'contingentemente' " (Walton, 2014: 247).

Estos sucesos fortuitos e incalculables, las enfermedades, la muerte repentina, la violencia y muchos otros entre los que se encuentra el nacimiento, son temas que Husserl aborda desde la metafísica, porque son problemas de la realidad fáctica. El cuerpo ocupa un lugar especial en la metafísica de Husserl y de ello quiero hablar en el siguiente apartado.

\section{CUERPO PROPIO Y METAFÍSICA}

Que la subjetividad trascendental se materialice en la realidad a través del cuerpo constituye uno de los mayores problemas de la fenomenología trascendental. ¿Cómo se da este entrelazamiento entre el cuerpo y el sujeto trascendental o conciencia? ¿Cómo es posible que la conciencia trascendental, que no es una parte del mucho, que es anterior al mundo, aparezca en el mundo como uno más de los facta del mundo? A mi juicio, se trata de un problema que ya no le concierne a la filosofía primera sino a la metafísica. Expongo a continuación las razones que sustentan esta tesis.

Husserl vio la fenomenología trascendental en el sentido de una ontología universal o fenomenología eidética, lo que constituye la filosofía primera, y lo que allí llama ciencia de los facta para referirse a la metafísica o filosofía segunda [Hua IX, 299]. El objetivo principal de la filosofía primera sería constituirse en el fundamento de todas las ciencias que se dirigen de un modo u otro a los objetos. Por esta razón, la fenomenología trascendental no solo es ontología universal, sino que alcanza en Husserl el sentido de filosofía primera (erste Philiosophie). La conciencia trascendental o subjetividad trascendental -misma que hay que entender como intersubjetividad trascendental- es el fundamento de toda trascendencia. Esto es, cualquier objeto que se constituye como trascendencia desde la conciencia trascendental, objetos que estudian las ontologías regionales, tiene justamente como fundamento, es decir, como fuente última de sentido y validez, la subjetividad trascendental que está detrás (Husserl, 2013: 109 [Hua III/I, 193]).

Frente a la fenomenología trascendental entendida como ontología universal o filosofía primera, se encuentra la metafísica o filosofía segunda. La metafísica se encargaría del estudio de la realidad o, en otras palabras, 
sería la "ciencia fundamental de la realidad" como afirma L. Tengelyi. Se trata de una metafísica de la facticidad y tendría como tema u objeto aquello que es dado como factum, es decir, como hecho del mundo. No se trata de algo que es dado de modo absoluto. Se trataría del sentido de la subjetividad trascendental vista como factum detrás del cual ya no sería posible retroceder.

Pues bien, además de un análisis constitutivo del cuerpo como instancia en la que se da este entrelazamiento con la conciencia, alma o subjetividad trascendental, el cual se realiza desde una perspectiva trascendental, es posible abordar el tema del cuerpo como problema metafísico. En su artículo sobre Teleología Husserl empieza enfatizando el problema de la "autoconstitución de la subjetividad" y habla del ser de la subjetividad que se encuentra en "contradicciones siempre nuevas" (Husserl, 1997: 6) y una de esas contradicciones, radica en el hecho de que la subjetividad trascendental se descubre "existiendo en la forma, que le es necesaria, de la mundanidad". Este es un caso único y notable, afirma Husserl y se refiere con ello a la relación que hay "entre el factum y el eidos". "El ser del eidos, el ser de las posibilidades eidéticas y del universo de estas posibilidades, es libre con respecto al ser o no ser de una u otra realización de tales posibilidades: es independiente de toda realidad — de toda realidad que le corresponde, se entiende. En cambio, el eidos yo trascendental es impensable sin el yo trascendental como yo fáctico" (Husserl, 1997: 12). A nuestro juicio el yo trascendental solo puede ser tal a través del cuerpo vivo en el que se concretiza y aparece en el mundo. Pero que aparezca como cuerpo en el mundo, como hecho que se da en un momento histórico determinado, significa que en algún momento no estaba, que el sujeto trascendental como cuerpo tuvo que nacer un día determinado y que, sin saber cuándo ni cómo, un día también tiene que morir. El nacimiento y la muerte del sujeto trascendental que está esencialmente entrelazado con el cuerpo, son fenómenos que están más allá de la constitución originaria del yo. Es decir, lo que sabemos de nuestro nacimiento lo sabemos gracias a los otros; otro nos pone un nombre, nos corta el ombligo, el cuerpo mismo tiene huellas o cicatrices de accidentes que vivió en determinado momento, pero de los cuales no sabemos nada. Por ello Husserl se refiere al nacimiento y a la muerte como fenómenos o problemas límite o de un nivel superior que deben ser abordados desde la metafísica.

De acuerdo con Husserl sin los "facta primordiales de la hyle... ningún mundo sería posible, ni ninguna subjetividad trascendental global" (Husserl, 1997: 13). Si esto es así, entonces la subjetividad trascendental necesita de la materia, del cuerpo, para constituirse como factum, esto es, como hecho del mundo. Pero no se trata de cualquier hecho, sino de un hecho que Husserl llama "factum primordial". 
Las líneas hacia donde hay que dirigir las siguientes reflexiones sobre la subjetividad trascendental como el factum primordial y su relación con el cuerpo, sigue siendo un tema actual y difícil de abordar y sobre el cual es necesario seguir reflexionando.

\section{COLOFÓN}

En los apartados anteriores hemos abordado el problema del cuerpo vivo o propio desde la fenomenología trascendental desde varias facetas que se complementan. En primer lugar, vimos que el cuerpo propio es desconectado y puesto entre paréntesis en Ideas I y que Husserl no le dedica un análisis minucioso y exhaustivo en esta obra que se despliega a partir de una fenomenología estática. El cuerpo del ser humano empírico es, como cualquier otra cosa del mundo, constituido por la subjetividad trascendental; aparece entonces como una unidad intencional con un sentido intencional que es correlativo a la conciencia. En esta obra, es decir, en Ideas I Husserl anticipa el problema del entrelazamiento del cuerpo y la conciencia y lo relaciona con la vida animal, pero no lo desarrolla y al no hacerlo podríamos caer en el error de pensar que la subjetividad trascendental es incorpórea.

En segundo lugar, revisamos los parágrafos que Husserl dedica a la constitución del cuerpo en Ideas II. Los análisis contenidos allí se encuentran en el horizonte de una fenomenología genética que no está separada de la estática, sino que se complementan. Allí Husserl dedica importantes reflexiones al cuerpo vivo, propio o animal y describe aspectos fundamentales de su constitución. Entre ellos se encuentra en primer lugar el hecho de que el cuerpo vivo de constituye como cuerpo vivo (Leib) y como cuerpo material o físico (Körper), pero sin ser nunca solo cuerpo material y no lo es porque las ubiestesias, es decir, las sensaciones localizadas constituyen el cuerpo vivo en tanto que cuerpo vivo que siente. El cuerpo es, por tanto, el portador de sensaciones localizadas en determinadas partes 0 zonas del cuerpo. Éste también se constituye como instrumento de la libre voluntad -lo cual abre un horizonte importante para la ética- y es un órgano de libre movimiento que permite una constitución dinámica del mundo; es, además, el punto cero de las coordenadas espaciales y a pesar de que es a partir del cuerpo como se constituye el mundo y cómo se aparecen las cosas en la cercanía y la lejanía, arriba y abajo, etc., el cuerpo propio mismo resulta inaccesible para sí, o no al menos de forma absoluta. El cuerpo no se puede percibir a sí mismo de forma originaria y hay zonas que le resultan inaccesibles.

En el tercer apartado tomamos como punto de partida el análisis de las ubiestesias y de las cinestesias y cenestesias - como las llama Serrano 
de Haro y San Martín - y apoyándonos en los trabajos de R. Walton exploramos algunas relaciones entre las cinestesias (por tanto, el cuerpo), los valores sensibles y los valores espirituales, lo que sirve de punta de lanza para adentrarse al estudio de la ética fenomenológica de Edmund Husserl.

La introducción de la ética y los valores constituidos a partir de la experiencia corporal dio paso al análisis de algunos problemas de la metafísica, a problemas de la contingencia, lo irracional del mundo y los sucesos inesperados que irrumpen en los proyectos universales del hombre ético, y de allí pasamos a problema de la subjetividad trascendental como factum, como hecho. Que la subjetividad trascendental requiera necesariamente (y no accidentalmente) del cuerpo vivo para concretizarse y aparecer en el mundo, pero el hecho mismo de su aparición, el nacimiento y la muerte, son problemas que deben abordarse desde la metafísica.

\section{REFERENCIAS}

-Gaos, J. (1997). La Lebenswelt de Husserl. En A. Serrano de Haro (Ed.), La posibilidad de la fenomenología (pp. 327-331). Madrid: Universidad Complutense.

-Husserl, E. (1968). Pbänomenologische Psychologie. Vorlesungen Sommersemester 1925. [Hua IX. Edited by Walter Biemel. The Hague/Netherlands: Martinus Nijhoff].

-Husserl, E. (1973). Zur Phänomenologie der Intersubjektivität. Texte aus dem Nachlass. Erster Teil. 1905-1920. [Hua XIII. Edited by Iso Kern. The Hague/Netherlands: Martinus Nijhoff].

-Husserl, E. (1997). Teleología (A. Serrano de Haro, Trad.). Daimon. Revista de Filosofía, (14), 5-14.

-Husserl, E. (2005). Ideas relativas a una filosofía fenomenológica y una filosofía trascendental, Libro II: Investigaciones fenomenológicas sobre la constitución. (A. Zirión, Trad.). México: FCE/UNAM. [Hua IV. Ideen zu einer reinen Pbänomenologie und phänomenologischen Philosophie. Zweites Bucb: Phänomenologische Untersucbungen zur Konstitution. Martinus Nijhoff, La Haya, 1952].

-Husserl, E. (2009). Las conferencias de París. Introducción a la fenomenología trascendental. (A. Zirión, Trad.). México: UNAM. [Hua I. Cartesianische Meditationen und Pariser Vorträge. Edited by S. Strasser. The Hague, Netherlands: Martinus Nijhoff, 1973].

-Husserl, E. (2013). Ideas relativas a una fenomenología pura y una filosofía fenomenológica. Libro primero: Introducción general a la fenomenología pura (nueva edición y refundición integral de la traducción de J. Gaos que abarca los dos volúmenes, por A. Zirión). México: UNAM/FCE. [Hua III/I. Ideen zu einer reinen Phänomenologie und phänomenologischen Pbilosophie. Erstes Buch: Algemeine Einfhürun in die reine Phänomenologie. Zweites Buch: Ergänæende Texte (19121929), ed. de Karl Schuhmann, La Haya: M. Nijhoff, 1976].

-Merleay-Ponty, M. (1975). Fenomenología de la percepción. (J. Cabanes, Trad.). Barcelona: Península. 
-San Martín, J. (2015a). La nueva imagen de Husserl. Lecciones de Guanajuato. Madrid: Trotta.

-San Martín, J. (2015b). Antropología filosófica II. Vida humana, persona y cultura. Madrid: UNED.

-Stein, E. (2004). Sobre el problema de la empatía. (J. L. Caballero Bono, Trad.). Madrid: Trotta.

-Waldenfels, W. (2015). Exploraciones fenomenológicas acerca de lo extraño. (Edición de Gustavo Leyva). Barcelona/México: Anthropos/UMSNH.

-Walton, R. (2014). Facetas de la corporalidad en la ética husserliana. Eidos, (21), 237-259.

-Walton, R. (2015). Intencionalidad y horizonticidad. Bogotá: Aula de Humanidades.

Sumario: Introducción; 1. El problema del cuerpo en Ideas I; 2. La constitución del cuerpo propio en Ideas II; 3. Notas para una valoración ética del cuerpo; 4. Cuerpo propio y metafísica; 5. Colofón; Referencias. 